Arq. Bras. Med. Vet. Zootec., v.66, n.3, p.721-730, 2014

\title{
Distribuição espacial de acidentes escorpiônicos em Belo Horizonte, Minas Gerais, 2005 a 2009
}

\author{
[Spatial distribution of scorpion sting accidents in Belo Horizonte, \\ Minas Gerais, 2005 to 2009] \\ A.D. Barbosa ${ }^{1}$, J.A. Silva ${ }^{1}$, M.F.E.C. Cardoso ${ }^{2}$, J.N.C. Meneses ${ }^{1}$, M.C.M. Cunha ${ }^{3}$, \\ J.P.A. Haddad ${ }^{1}$, R.R. Nicolino ${ }^{1}$, D.F. Magalhães ${ }^{1}$ \\ ${ }^{1}$ Escola de Veterinária - Universidade Federal de Minas Gerais - Belo Horizonte, MG \\ ${ }^{2}$ Unidade de Toxicologia - Hospital João XXIII - FHEMIG - Belo Horizonte, MG \\ ${ }^{3}$ Secretaria Municipal de Saúde de Belo Horizonte - Belo Horizonte, MG
}

\begin{abstract}
RESUMO
Acidentes por escorpião constituem problema de saúde pública em Belo Horizonte. Realizou-se um estudo epidemiológico observacional retrospectivo para analisar a frequência e distribuição espacial dos acidentes escorpiônicos em Belo Horizonte, entre 2005 e 2009, e associá-las às categorias de risco classificadas pelo Índice de Vulnerabilidade à Saúde (IVS), um índice socioeconômico regional composto. Foram utilizados dados de notificação do Sistema Nacional de Agravos de Notificação (SINAN) e Sistema de Vigilância Epidemiológica (SISVE), referentes aos anos de 2008 e 2009. Para o período de 2005 a 2007, foi necessário o resgate de dados das fichas clínicas do Hospital João XXIII (HPSJXXIII) correspondentes às variáveis encontradas no SINAN e SISVE, uma vez que os mesmos não estavam digitalizados nos referidos sistemas de informação. Para georreferenciamento e análise espacial, foi utilizada a base geográfica EndGeo, o aplicativo de mapeamento MapInfo versão 10.0 e os programas Hotspot Detective e SatScan. Entre 2005 e 2009, ocorreram em Belo Horizonte 2.769 casos de acidentes por escorpião, o que representa uma incidência média de 22,4 casos por 100.000 habitantes. Do total de casos, $1.924(69,5 \%)$ foram georreferenciados e houve predomínio de incidência em dois Distritos Sanitários (DS), com grande concentração de casos nas regiões de cemitérios do município. Foram detectados dois "clusters" no período, sendo um em 2005, nos DS Noroeste e Oeste, e outro entre 2006 e 2007, nos DS Noroeste e Nordeste. Não houve associação entre as áreas de maior incidência de escorpionismo e as áreas de maior risco à saúde classificadas pelo IVS. Com base nos resultados, concluise que há necessidade de melhorias no processo de notificação do agravo e que o mapeamento dos casos é uma ferramenta relevante capaz de embasar o direcionamento das ações educativas de controle para as áreas prioritárias de Belo Horizonte.
\end{abstract}

Palavras-chave: acidentes por escorpião, distribuição espacial, índice de vulnerabilidade à saúde

\begin{abstract}
Scorpion sting accidents are a public health problem in Belo Horizonte and veterinarians play an important role in its control program when based on capture, mapping of cases and educational actions with the population. A retrospective epidemiological study was done to analyze the frequency and spatial distribution of scorpion sting cases in Belo Horizonte, between 2005 and 2009, and to associate them with the Health Vulnerability Index (IVS), a regional and composed socio-economic index. Notification data of scorpion sting accidents were used from the National System of Notifiable Diseases (SINAN) and Epidemiological Surveillance System (SISVE) for years 2008 and 2009. For the period between 2005 and 2007 it was necessary to retrieve data from the clinical records of João XXIII Hospital (HPSJ XXIII) which were not digitalized in the referred information systems. For georeference and spatial analysis, geographic base EndGeo and features of the programs MapInfo version 10.0, Hotspot Detective and
\end{abstract}

Recebido em 6 de agosto de 2013

Aceito em 1 de novembro de 2013

E-mail:amandaduarte@ufmg.br 
SatScan were used. Between 2005 and 20092769 cases of scorpionism, occurred in Belo Horizonte which represents an average incidence of 22.4 cases per 100,000 inhabitants. Among 1924 (69.5\%) georeferenced cases, the highest incidence was observed in two Sanitary District (DS). A large concentration of cases was also observed in the areas of the city cemeteries. Two clusters were detected during this period, one in 2005, located in the Northwest and West DS, and another between 2006 and 2007 in the Northwest and Northeast DS. There was no association between higher incidence areas of scorpionism in Belo Horizonte and the areas of higher health risk classified by IVS. It is necessary to improve the reporting process regarding scorpion sting accidents. Another conclusion is that the mapping of cases is a relevant tool to base the targeting of educative actions to the priority areas of Belo Horizonte.

Keywords: scorpion sting accidents, spatial distribution, Belo Horizonte, health vulnerability index

\section{INTRODUÇÃO}

Os escorpiões são artrópodes que predominam nas zonas tropicais e subtropicais do mundo. Quatro espécies do gênero Tityus têm sido responsabilizadas por acidentes humanos no Brasil: Tityus serrulatus, Tityus bahiensis, Tityus stigmurus e Tityus paraensis, sendo o $T$. serrulatus responsável pela maioria dos casos mais graves (Manual..., 2009). Essa espécie tornou-se bem adaptada à vida domiciliar urbana, com grandes possibilidades de abrigos e uma alimentação farta (Bucherl, 1979). Além disso, possui o veneno mais potente e pode se reproduzir por partenogênese, o que facilita ainda mais sua proliferação (Manual..., 2009).

Segundo Chippaux e Goyffon (2008), os escorpiões são responsáveis por 1,2 milhões de acidentes e por cerca de 3.250 óbitos no mundo anualmente. No Brasil, os acidentes por animais peçonhentos hoje representam mais de 113 mil notificações e 299 óbitos por ano, sendo que, dentre eles, o acidente escorpiônico tem se destacado pela sua alta e crescente magnitude (Manual..., 2009). Acidente escorpiônico ou escorpionismo é o quadro de envenenamento humano provocado pela inoculação de veneno através de aparelho inoculador (ferrão ou télson) de escorpiões. Sua importância é acrescida, de acordo com a espécie causadora, pela ocorrência urbana e precocidade da evolução fatal na faixa etária pediátrica (Situação..., 2010). Em 2009, foram notificados no Brasil 45.721 acidentes causados por escorpiões, representando um aumento superior a sete mil casos quando comparado ao ano anterior (38.671) (Situação..., 2010). Os casos de escorpionismo no país são relatados em quase todos os Estados, principalmente em Minas Gerais e São Paulo (Soares et al., 2002; Cupo et al., 2003).
Ao causar óbitos ou sequelas temporárias que impossibilitem o trabalho, o escorpionismo deve ser objeto constante de ações públicas, visando à prevenção dos acidentes (Vigilância..., 2009). Para isso, é de alta relevância o conhecimento da epidemiologia do agravo, especialmente da sua distribuição espacial, para auxiliar no direcionamento das políticas de saúde.

Objetivou-se com este trabalho verificar a frequência e distribuição espacial dos acidentes escorpiônicos em Belo Horizonte, de 2005 a 2009, e associá-las às categorias de risco classificadas pelo Índice de Vulnerabilidade à Saúde (IVS), um índice socioeconômico regional composto.

\section{MATERIAL E MÉTODOS}

A área de estudo foi o município de Belo Horizonte, capital do estado de Minas Gerais, localizado na região sudeste do Brasil. Possui clima predominantemente tropical, com média de temperatura anual de $20,5^{\circ} \mathrm{C}$. Sua população humana variou de 2.374.125 habitantes em 2005 a 2.452.617 habitantes em 2009, sendo a população média para o período de 2.414.848 habitantes (Estimativas..., 2010). A área territorial do município é de $331 \mathrm{~km}^{2}$, dividida em nove regiões administrativas com autonomia financeira e gerencial segundo a Prefeitura Municipal (Regionais..., 2008): Barreiro, CentroSul, Leste, Nordeste, Noroeste, Norte, Oeste, Pampulha e Venda Nova.

Realizou-se um estudo epidemiológico observacional retrospectivo a partir de uma demanda da Secretaria Municipal de Saúde da Prefeitura de Belo Horizonte (SMSA-PBH) em resolver problemas relacionados a deficiências no processo de notificação e mapeamento dos 
casos de acidentes por escorpião no município, os quais dificultavam o desencadeamento eficaz das ações de controle. $\mathrm{O}$ estudo foi feito segundo a metodologia utilizada por Campolina (2006) em sua pesquisa, a partir de dados do Sistema de Informação de Agravos de Notificação (SINAN, 2010), e Sistema de Vigilância Epidemiológica (SISVE, 2009). O SISVE foi desenvolvido especificamente para o município de Belo Horizonte e permite a localização espacial e geográfica do logradouro onde ocorreu o acidente, com recursos do Sistema de Apoio ao Georreferenciamento (SISGEO) e das bases geográficas da PRODABEL (Empresa de Informática e Informação do Município de Belo Horizonte S/A). Os bancos SINAN/SISVE, de posse da Gerência de Epidemiologia e Informação da Secretaria de Saúde da Prefeitura de Belo Horizonte (SMSA/BH), são compostos de dados coletados no Serviço de Toxicologia do Hospital João XXIII (HPSJXXIII), da Fundação Hospitalar de Minas Gerais.

Foi necessária a recuperação de dados das fichas clínicas de atendimento a vítimas de acidentes por escorpião do Setor de Toxicologia do HPSJXXIII, no período de 2005 a 2007, uma vez que a grande maioria delas ainda não se encontrava digitalizada nos sistemas SINAN e SISVE, no momento do estudo. Além de dados referentes ao local do acidente, as fichas clínicas continham informações a respeito da idade e profissão do paciente, local da picada, espécie do escorpião, ambiente de exposição, tempo entre picada e atendimento, manifestações clínicas, soroterapia e evolução do quadro clínico. Os dados foram organizados no programa Excel versão 2003.

Para análise espacial, foram obtidas, primeiramente, as coordenadas " $x$ " e " $y$ " para os endereços recuperados no HPSJXXIII. Essa geocodificação foi feita a partir do cruzamento desses dados com a tabela de dados da base geográfica EndGeo, gentilmente cedida pela PRODABEL, a qual contém as coordenadas geográficas dos endereços de Belo Horizonte. Para isso, foi utilizado o programa MapInfo Professional versão $10.0 \quad$ (SN MINWBU1000005149O). Para os endereços que não foram geocodificados automaticamente pelo programa, foi realizada consulta manual às bases geográficas EndGeo. No caso de números não encontrados com exatidão nas bases, foi feita uma aproximação de até 100 números a mais ou 100 números a menos, assim como é feito pela SMSA através do sistema SISVE.

Os casos de escorpionismo em Belo Horizonte foram então distribuídos espacialmente em mapas de acordo com os DS do município, Áreas de Abrangência, áreas verdes, rios e cemitérios. Em seguida, foram analisados estatisticamente com a utilização de técnicas de geoprocessamento. Para visualização geral da intensidade do processo em todas as regiões dos mapas, utilizou-se a técnica de densidade por Kernel, com os recursos do programa "Hotspot detective" para MapInfo. Para a verificação das áreas do município onde o número de casos encontrava-se acima do esperado, foi utilizado o método estatístico Space-Time Permutation Model para detecção de "clusters" (Kulldorff $e t$ al., 2005). Essa análise foi feita com auxílio do software SatScan.

Foi confeccionado também um mapa temático para representar a frequência e distribuição dos casos de acidentes escorpiônicos de acordo com as categorias de risco classificadas pelo Índice de Vulnerabilidade à Saúde (IVS). O IVS, elaborado pela Gerência de Epidemiologia e Informação - GEEPI, órgão da estrutura administrativa da Secretaria de Saúde do Município de Belo Horizonte, é uma associação de variáveis socioeconômicas (saneamento, habitação, educação, renda) e sociais/saúde, ponderadas de acordo com pesos e indicadores específicos (Índice... 2003). A aplicação do IVS na população humana de Belo Horizonte, estimada em 2.238.322 para o ano de 2003, mostrou que $627.224(28,02 \%)$ pessoas estavam em áreas consideradas de risco baixo, 849.611 $(37,96 \%)$ em áreas de risco médio, 603.600 $(26,96 \%)$ em áreas de risco elevado e 157.897 $(7,05 \%)$ em áreas de risco muito elevado.

\section{RESULTADOS E DISCUSSÃO}

Entre 2005 e 2009, ocorreram em Belo Horizonte 2.769 casos de acidentes por escorpião, o que representa uma incidência média de 22,4 casos/100.000 habitantes e uma incidência acumulada de 114,7 casos por 100.000 habitantes. Do total de casos, 1.924 (69,5\%) foram georreferenciados. 
Em relação à qualidade do georreferenciamento, verificou-se que, do total de endereços, $47,35 \%$ foram exatamente localizados, $22,14 \%$ tiveram o número do imóvel aproximado, 13,29\% não foram encontrados nas bases geográficas e em $17,22 \%$ dos casos, cujos dados foram resgatados de prontuários do HPSJXXIII, faltavam informações como nome do logradouro e número do imóvel, necessárias para o processo de geocodificação.

Ao se analisar a distribuição espacial dos casos de acidente escorpiônico, observou-se que a porcentagem de perdas do georreferenciamento foi relativamente alta, quando comparada às perdas observadas em outros estudos, como os de Nunes et al. (2000) e Campolina (2006). Um fato relevante foi a discrepância entre os períodos de 2005 a 2007 e de 2008 a 2009, no que diz respeito ao número de casos com endereço "não encontrado" ou "sem informação". Observou-se que essas perdas foram bem menores considerando-se o banco do SINAN/SISVE. Isso leva à constatação de que a digitalização sistemática, feita pela Prefeitura de Belo Horizonte quando os dados são adequadamente notificados, melhora a qualidade da informação espacial gerada.

Analisando-se os 1.924 casos georreferenciados e sua distribuição por DS de Belo Horizonte, calculou-se a incidência acumulada para o período de 2005 a 2009, por Regional. O DS Noroeste foi o que apresentou maior incidência acumulada (132,2 casos/100.000 habitantes), seguido dos DS Nordeste (126,7 casos/100.000 habitantes), DS Norte (100,01 casos/100.000 habitantes), DS Pampulha (89,76 casos/100.000 habitantes), DS Venda Nova $(75,93$ casos/100.000 habitantes), DS Leste $(68,27$ casos/100.000 habitantes), DS Oeste $(65,64$ casos/100.000 habitantes), DS Centro-Sul (61,8 casos/100.000 habitantes) e DS Barreiro (20,21 casos/100.000 habitantes). Na Figura 1, é possível observar que houve poucos casos de acidente por escorpião nas áreas verdes e, ao contrário, uma grande concentração de casos nos cemitérios e suas proximidades.

Ao se aplicar a técnica de densidade por Kernel no mapa, pode-se observar que, além da maior intensidade do agravo nos DS Noroeste e Nordeste, principalmente na área limítrofe entre as duas Regionais, há também um ponto de alta concentração de acidentes no DS Oeste (Fig. 2).

Pela análise Space-Time-Permutation, foram detectados dois "clusters" no período estudado. Nesse teste, analisando-se a distribuição espaçotemporal do escorpionismo em intervalos de 1 ano, detectou-se um cluster em 2005 ( $\mathrm{p}=0,04)$, com 12,28 casos esperados e 32 observados, localizado entre os DS Noroeste e Oeste. Já na análise em intervalos de 2 em 2 anos, foram observados o mesmo "cluster" do ano de 2005 e outro entre 2006 e 2007 ( $\mathrm{p}=0,016)$, com 72,34 casos esperados e 107 observados, o qual está localizado em uma área entre os DS Noroeste e Nordeste (Fig. 3).

A maior incidência encontrada nos Distritos NE e NO também foi verificada por Nunes et al. (2000) e Campolina (2006). Como possíveis determinantes para o aparecimento e ocorrência de acidentes escorpiônicos nessas regiões e também no DS Oeste do município, como demonstrado pelas técnicas de Kernel e de detecção de "clusters", devem ser considerados fatores como presença de casas antigas, domicílios vagos, cemitérios, pedreiras, imóveis abandonados, baixo índice de crescimento vertical em relação ao crescimento horizontal, presença de favelas e condições precárias de higiene e saneamento (Plano... 1995; Anuário, 2003; Campolina, 2006).

Os períodos em que foram detectados os "clusters" (2005 e 2006-2007) podem ser explicados por uma possível deficiência no sistema de notificação dos acidentes ao SINAN nos anos 2008 e 2009, ou até mesmo por possíveis melhorias nas ações de controle do agravo a partir de 2008 (Barbosa, 2011). 


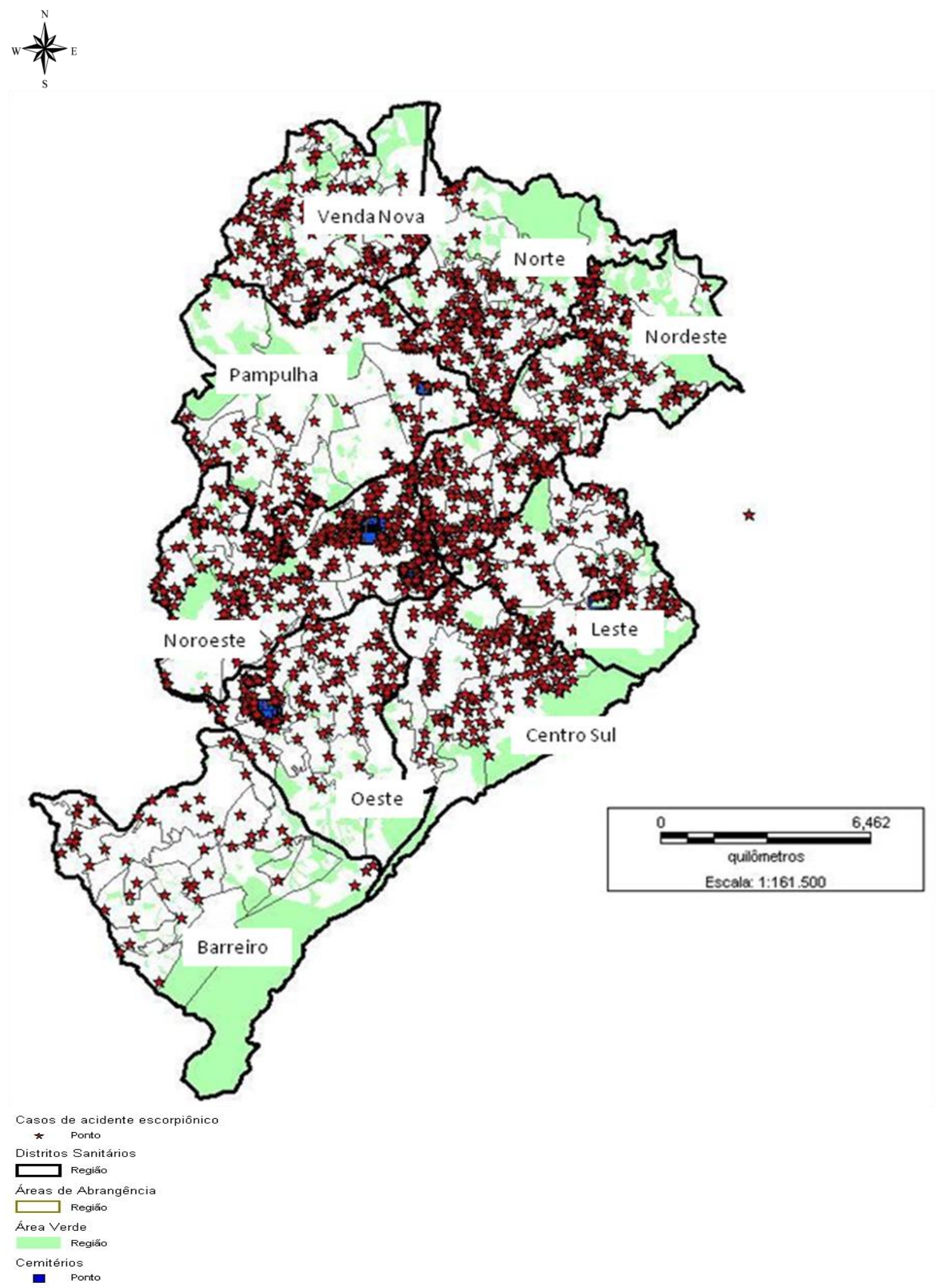

Figura 1. Casos de acidente escorpiônico por Área de Abrangência, Distrito Sanitário, localização de cemitérios e áreas verdes, em Belo Horizonte, entre 2005 e 2009. 


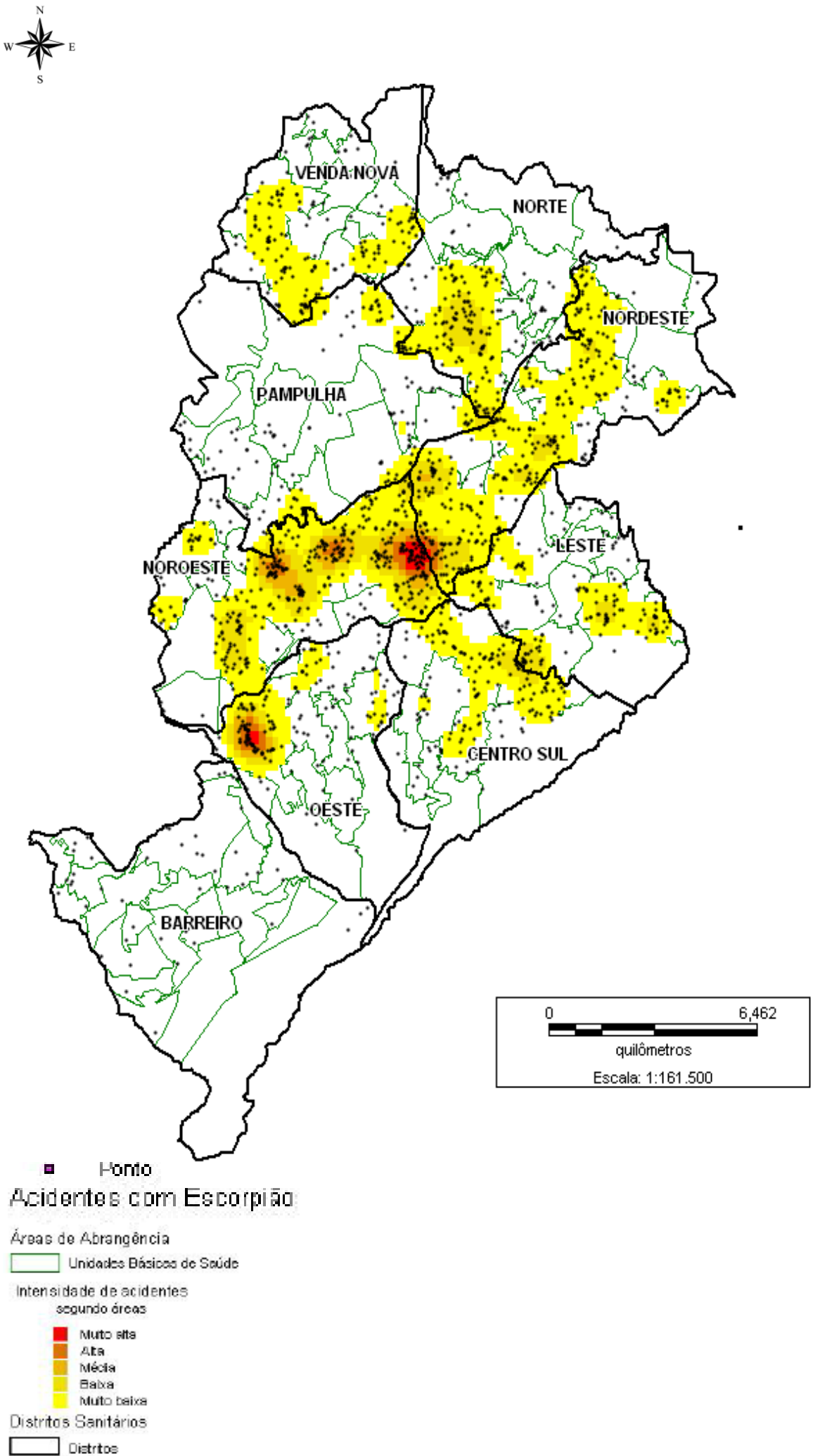

Figura 2. Distribuição e intensidade dos casos de acidente escorpiônico de acordo com as Áreas de Abrangência e os Distritos Sanitários de Belo Horizonte, 2005 a 2009. 


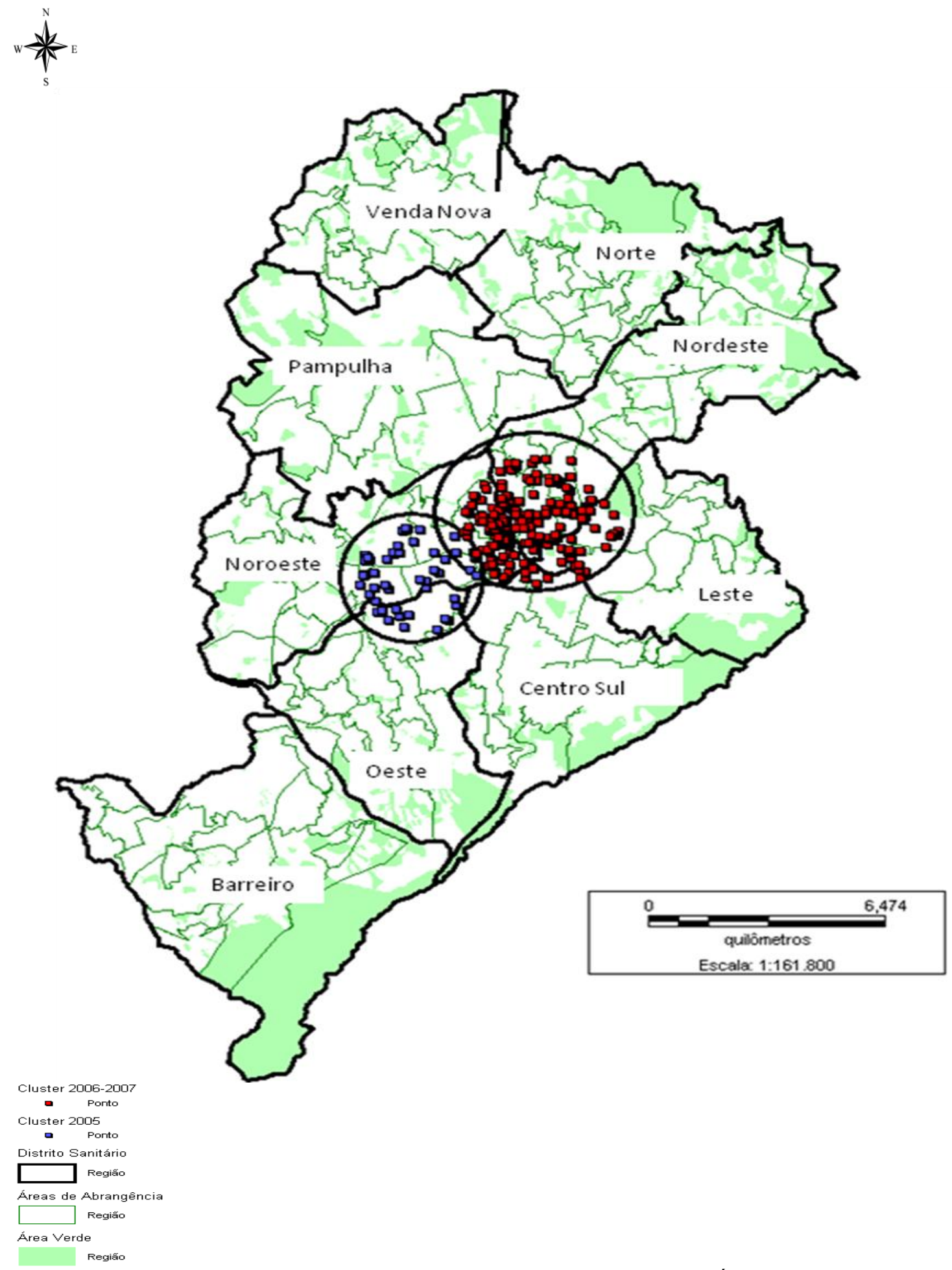

Figura 3. "Clusters" de casos de acidente escorpiônico de acordo com as Áreas de Abrangência e os Distritos Sanitários, em Belo Horizonte, 2005 e 2006 - 2007. 


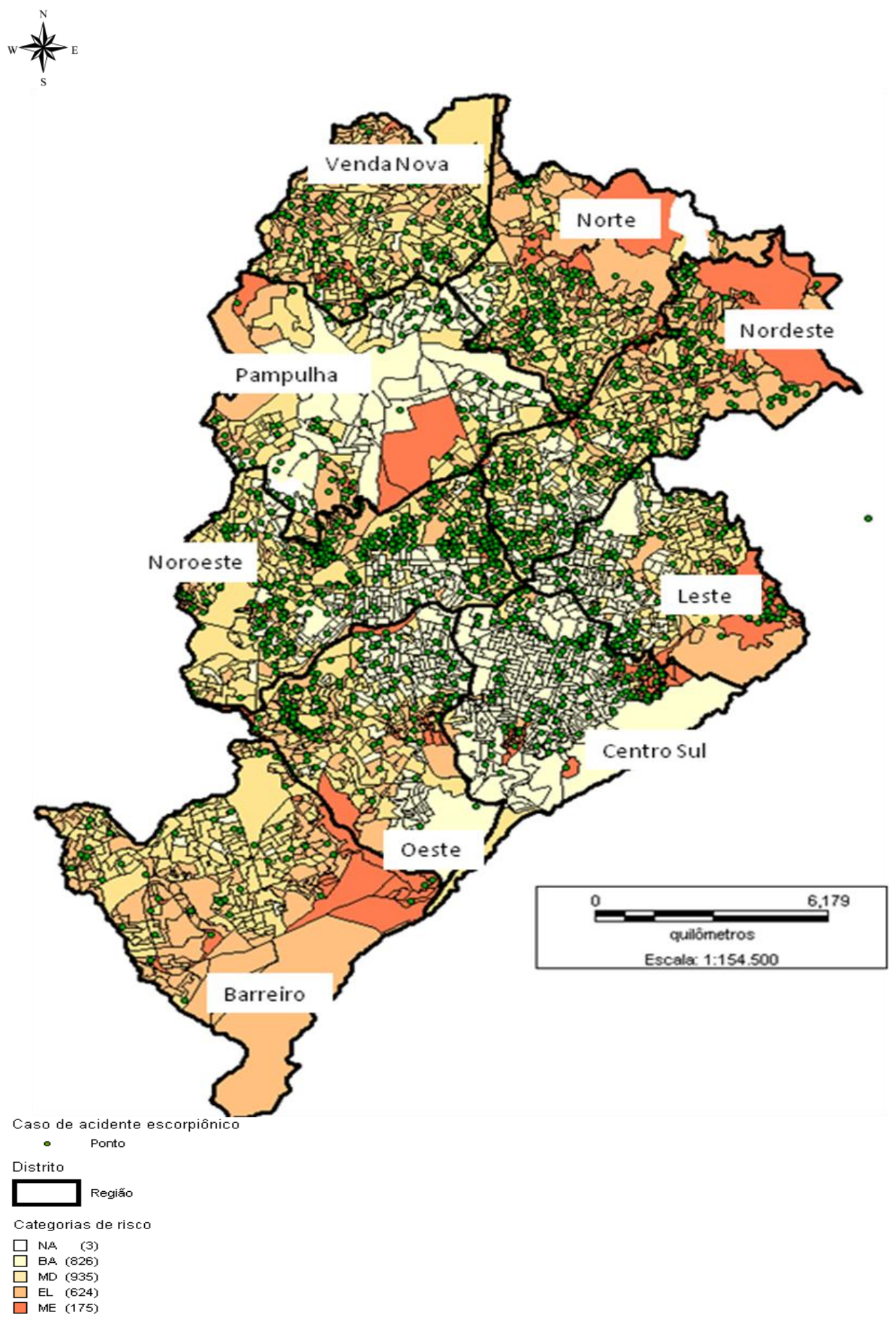

Legenda: $\mathrm{NA}=$ não aplicável, $\mathrm{BA}=$ baixo, $\mathrm{MD}=$ médio, $\mathrm{EL}=$ elevado, $\mathrm{ME}=$ muito elevado.

Figura 4. Casos de acidente escorpiônico de acordo com as Áreas de Risco classificadas pelo Índice de Vulnerabilidade à Saúde, em Belo Horizonte, entre 2005 e 2009. 
Tabela 1. Distribuição e incidência dos casos de acidente escorpiônico, por 10.000 habitantes, de acordo com a categoria de risco classificada pelo Índice de Vulnerabilidade à Saúde, em Belo Horizonte, no período de 2005 a 2009

\begin{tabular}{lcccc}
\multirow{2}{*}{ Categoria de risco } & População exposta & \multicolumn{3}{c}{ Casos de acidentes escorpiônicos } \\
\cline { 3 - 5 } & & & $\%$ & $\begin{array}{c}\text { Frequência por } 10.000 \\
\text { habitantes }\end{array}$ \\
\hline Baixo & No. & 420 & 22,23 & $6,70 \mathrm{a}$ \\
Médio & 627.224 & 762 & 40,34 & $9,00 \mathrm{a}$ \\
Elevado & 849.611 & 551 & 29,17 & $9,13 \mathrm{a}$ \\
Muito Elevado & 603.600 & 156 & 8,26 & $9,88 \mathrm{a}$ \\
Total & 157.897 & 1889 & 100,00 & $8,44 \mathrm{a}$ \\
\hline
\end{tabular}

*Letras iguais em uma mesma coluna indicam igualdade estatística pelo teste do Qui-quadrado, com $\mathrm{p}>0,05$.

A não associação entre a distribuição dos casos e o IVS difere das conclusões de Spirandeli-Cruz et al. (1995), em trabalho realizado no município de Aparecida, São Paulo. Segundo esses autores, além das características ambientais próprias que favorecem o aparecimento de escorpiões, a incidência de casos em áreas urbanas periféricas de baixos níveis socioeconômicos e de saneamento é maior.

A partir dessas informações, uma hipótese que justificaria o resultado encontrado no presente estudo seria o fato de haver limitações do uso do IVS que, de acordo com os próprios criadores (Índice... 2003), incluem a possibilidade de perda de informação na construção de um indicador complexo, o mérito relativo de cada variável utilizada e a possível ausência de variáveis relevantes, como as geográficas e ambientais no caso do escorpionismo, por exemplo. Dessa forma, variáveis como saneamento e habitação, mais relacionadas ao aparecimento de escorpiões do que as demais variáveis utilizadas na construção do indicador, poderiam estar com o peso subestimado no que diz respeito a esse agravo especificamente. A alta porcentagem de informações perdidas no georreferenciamento também deve ser considerada como possível motivo para o resultado observado (Barbosa, 2011).

Novos estudos devem ser feitos para esclarecer os reais motivos da não associação encontrada entre a distribuição do escorpionismo em Belo Horizonte e as categorias de risco classificadas pelo IVS, testando-se hipóteses relacionadas aos fatores determinantes do agravo no município.

\section{CONCLUSÕES}

Há necessidade de melhorias no processo de notificação dos acidentes por escorpiões em Belo Horizonte para que sejam desencadeadas ações preventivas imediatas e, portanto, mais eficientes por parte do serviço público de saúde. O IVS não foi um bom indicador das áreas de maior incidência de acidentes escorpiônicos em Belo Horizonte, não devendo ser considerado para embasar a elaboração e aplicação de medidas de controle. Sugere-se considerar este estudo da distribuição espacial dos casos de escorpionismo para o planejamento das estratégias de controle do agravo no município, priorizando ações de educação em saúde e manejo ambiental nas áreas onde há maior ocorrência de acidentes (DS Nordeste, Noroeste e Oeste).

\section{AGRADECIMENTOS}

Agradecemos à Fundação de Amparo à Pesquisa do Estado de Minas Gerais pelo financiamento da pesquisa.

\section{REFERÊNCIAS}

ANUÁRIO estatístico de Belo Horizonte. 2003. Prefeitura de Belo Horizonte. Disponível em: <http://www.portal2pbh.pbh.gov.br>. Acessado em: 20 de novembro de 2008.

BARBOSA, A.D. Caracterização e distribuição espacial dos acidentes escorpiônicos em Belo Horizonte, Minas Gerais, 2005 a 2009. 44f. 2011. Dissertação (Mestrado em Ciência Animal) - Escola de Veterinária, UFMG, Belo Horizonte, MG. 
BUCHERL, W. Acúleos que matam. Rev. Tribunais, v.4, p.299-300, 1979.

CAMPOLINA, D. Georreferenciamento e estudo clínico-epidemiológico dos acidentes escorpiônicos atendidos em belo horizonte, no serviço de toxicologia de Minas Gerais. 127f. 2006. Dissertação (Mestrado em Medicina) Faculdade de Medicina, UFMG, Belo Horizonte, MG.

CHIPPAUX, J.P.; GOYFFON, M. Epidemiology of scorpionism: a global appraisal. Acta Tropical, v.107, p.71-79, 2008.

CUPO, P.; AZEVEDO-MARQUES, M.M.; HERING, S.E. Acidentes por animais peçonhentos: escorpiões e aranhas. Medicina, v.36, p.490-497, 2003.

ESTIMATIVAS de população. Brasília; Instituto Brasileiro de Geografia e Estatística, 2009. Disponível em: <http://www.ibge.gov.br> Acessado em: 17 set. 2010.

ÍNDICE de vulnerabilidade à saúde: gerência de epidemiologia e informação, 2003. Disponível em: <http://www.pbh.gov.br> Acessado em: 13 out. 2008.

KULLDORF, M.; HEFFERNAN, R.; HARTMAN, J. et al. A Space-Time permutation Scan Statistic for Disease Outbreak Detection. Plos Medicine, v.2, p.216-224, 2005.

MANUAL de controle de escorpiões. Brasília: Ministério da Saúde, 2009. 72p.

NUNES, C.S.; BEVILACQUA, P.D.; JARDIM, C.C.G. Aspectos demográficos e espaciais dos acidentes escorpiônicos no Distrito Sanitário Noroeste, Município de Belo Horizonte, Minas Gerais, 1993 a 1996. Cad. de Saúde Pub., v.16, p.213-223, 2000.
PLANO diretor de Belo Horizonte: lei de uso e ocupação do solo - estudos básicos. Belo Horizonte: Prefeitura Municipal de Belo Horizonte. 1995.

REGIONAIS. Prefeitura municipal de Belo Horizonte. Disponível em: 〈http://portal2.pbh.gov.br〉 Acessado em 10 de outubro de 2008.

SISTEMA de informação de agravos de notificação - SINAN. Brasília: DATASUS Ministério da Saúde, 2010. Disponível em: <http:tabnet.datasus.gov.br> Acessado em: 20 set. 2010 .

SITUAÇÃO epidemiológica das zoonoses de interesse para a saúde pública. SVS/MS. Boletim eletrônico epidemiológico. Ano 10, n.2, 2010. Disponível em: <www.saude.gov.br/svs > Acessado em: 27 set. 2010.

SOARES, M.R.M.; AZEVEDO, C.S.M.M. Escorpionismo em Belo Horizonte, MG: um estudo retrospectivo. Rev. Soc. Bras.Med. Tropical, v.35, p.359-363, 2002.

SPIRANDELI-CRUZ, E.F.S.; YASSUDA, C.R.W.; BARRAVIERA, J.J. Programa de controle de surto de escorpião Tityus serrulatus, Lutz e Mello 1922, no Município de Aparecida, São Paulo. Rev. Soc. Bras. Med. Tropical., v.28, p.123-128, 1995.

VIGILÂNCIA em saúde - zoonoses. Brasília; Ministério da Saúde, 2009. Disponível em: $<$ http://portal.saude.gov.br/portal/arquivos/pdf/ab cad22.pdf> Acessado em: 18 jan. 2009. 